\title{
Setworks: Networks of Complex Set Intersections
}

\author{
Alexander O. Savi ${ }^{1, *}$ \\ ${ }^{1} \mathrm{VU}$ University Amsterdam \\ *o.a.savi@gmail.com
}

\begin{abstract}
Sets are a universal concept in science. Visualizations of sets and their intersections contribute to an understanding of the complex relations that they comprise. Setworks capitalize on networks and bring set visualizations the power to scale and visualize increasingly complex, hierarchical, relations.
\end{abstract}

Science abounds with complex, often hierarchical, relations: phenotypes related to genetic variants, proteins related to tissue samples, error responses related to misconceptions, treatment effects related to experimental conditions, or responses to a pandemic related to governments. Such relations contribute to the massive complexity of systems throughout scientific disciplines, from biology to society. Sets can be used to represent these systems, where elements (phenotypes, proteins) are members of one or more sets (genetic variants, tissue samples) and consequently create intersections between sets.

The visualization of sets and their intersections aids the understanding of complex systems. The Venn diagram is arguably the best known and most widely used visualization, capturing the organization of two to four sets. However, visualizations struggle to capture many more sets, as the number of possible intersections grows exponentially with the number of sets $\left(2^{n}-n-1\right)$. As such, set visualizations demand trade-offs [1, such as in the number of considered sets and the depth of the intersections. Ultimately, these visualization limitations may put a limit to our understanding of complex relations.

Complex relational structures are typically visualized as networks. Networks reveal structure through nodes and links that represent individual elements and their relations. The power to reveal relational structure, combined with the scalability of networks, provides an opportunity for the visualization of sets and their intersections. Here, I introduce setworks: networks of complex set intersections. These include chord setworks and waterfall setworks. Crucially, their differential treatment of the inevitable trade-offs realizes very distinct features. The former conserves the individual elements, but at the cost of comprehensibility for intersections of three or more sets. The latter alleviates the aforementioned trade-off in the number of considered sets and the intersection depth, but at the cost of the individual elements. 
Figure 1 illustrates a chord setwork. In a chord setwork, nodes represent individual elements and are grouped by their sets in a circle. This is similar to a chord diagram, but in a chord setwork a node appears in every set of which it is a member, and may thus appear more than once. The edges connect the different copies of a node across sets. As such, edges connect intersecting sets. A node that is member of a single set is unconnected.

The chord setwork reveals various aspects of sets. It visualizes the proportion of unique elements in a set (the unconnected nodes) and the proportion of shared elements with one or more other sets (the connected nodes, differentiated by color). The chord setwork's most distinctive feature is the conservation of the individual elements. As the individual elements are not lost in the visualization, each element can be traced to the sets it belongs to. Although the chord setwork best transmits pairwise intersections, the number of edges connected to a particular node gives away the number of sets the element belongs to $(n+1)$, and those sets can be traced through the edges.

If pairwise intersections suffice, and the individual elements are not of interest, the nodes contained in a set and the edges contained in an intersection can be collapsed such that a pairwise setwork emerges. Effectively, this matches the network representation of a matrix or heatmap of pairwise intersections.

Then, Figure 2 illustrates a waterfall setwork. In a waterfall setwork, nodes represent sets and intersections and are hierarchically ordered by their intersection depth. Nodes on the top layer represent the sets, nodes on the second level represent their pairwise intersections, and so forth. Edges connect the intersection nodes with their parent nodes. Node size reflects the size of the set/intersection, whereas edge size reflects the size of the child node. The waterfall setwork is well suited for visualizing all intersections, but this comes at the cost of the individual elements.

The waterfall analogy aids in understanding the complex web of intersections. The sets at the top form the sources of the waterfall. From each of these sources, different streams cascade and combine into the lower layers. At each layer, the streams may form pools of intersections until ultimately the most complex intersection is reached. On top of revealing the sizes of individual sets and their intersections, the waterfall setwork provides a means to inspect their global relational structure. The edges, by means of their size and depth in the setwork, can reveal clusters of large sets with considerable overlap. And, by means of node size, each layer of the setwork shows the distribution of intersection size for a particular intersection depth. Also, waterfall setworks allow quick computations of some basic binary operations (e.g., the sum of two parent nodes minus their child node gives the size of the union of the parent nodes, the sum of two parent nodes minus twice their child node gives the size of the symmetric difference of the parent nodes).

Since setworks rely on networks, no dedicated software is required to create them. Both setworks in this article were created with ggraph [5], an open-source 
Figure 1: Chord setwork of governmental responses to the Covid-19 pandemic (in effect August 31, 2020). Nodes represent countries (elements) and are grouped and colored by their responses (sets). Responses include curfew (curfew implemented), domestic (domestic lockdown), restaurant (restaurants closed), and surveillance (mobile app or bracelet surveillance). Edges connect each country across their responses. Edge colors correspond to particular pairwise intersections between responses. Edges are bundled using hierarchical edge bundling [2]. Data source [3].

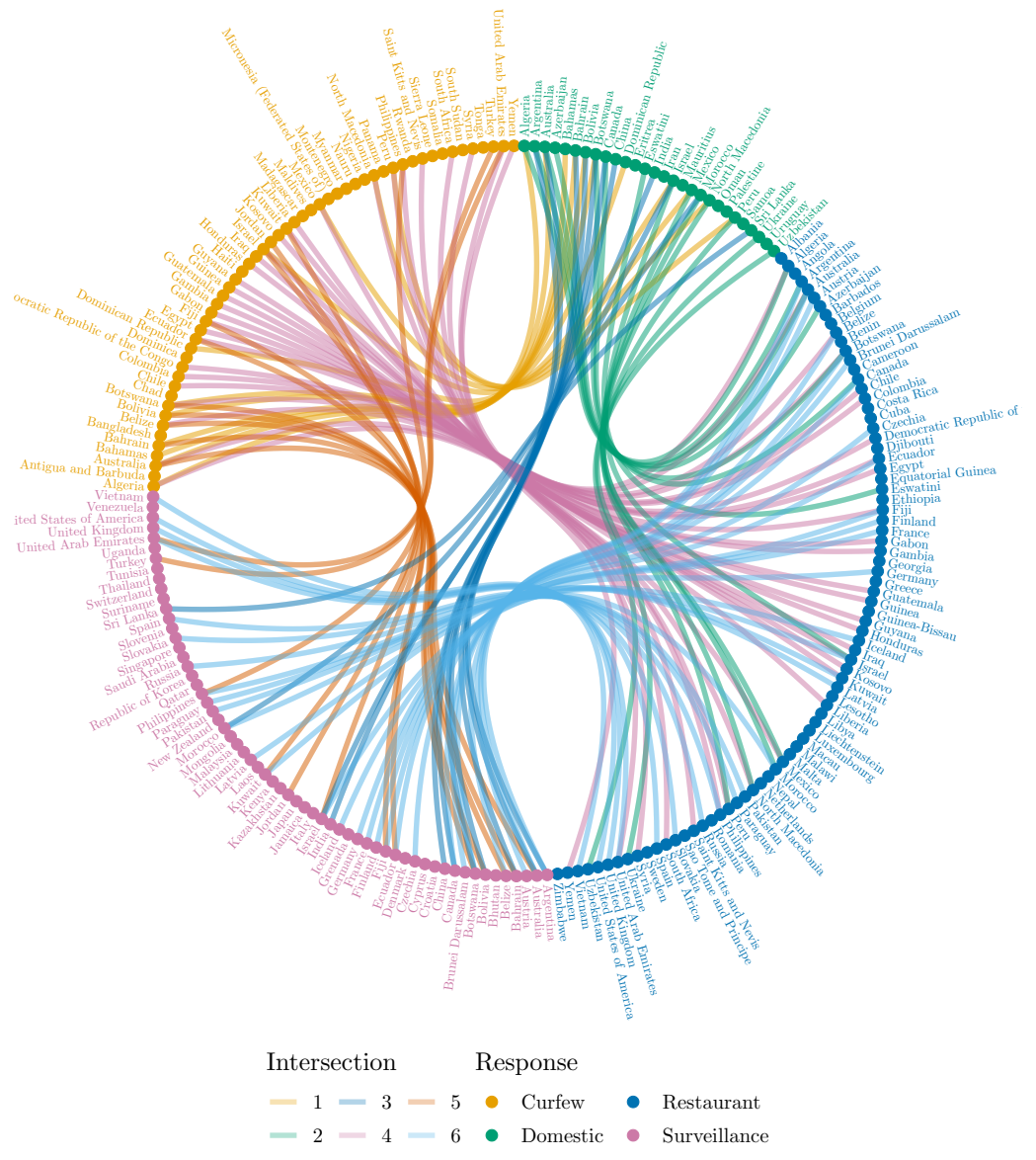


Figure 2: Waterfall setwork of governmental responses to the Covid-19 pandemic (in effect August 31, 2020). Nodes represent sets and intersections of governmental responses. Nodes are labeled with these responses: 1 (domestic lockdown), 2 (travel restrictions), 3 (curfew implemented), 4 (bans on mass gatherings), and 5 (bans on sporting and large events). Node size reflects the number of countries in a set/intersection. Edge size reflects the number of countries shared between the parent and child node. A layout for layered directed acyclic graphs is used [4]. Data source $[3$.

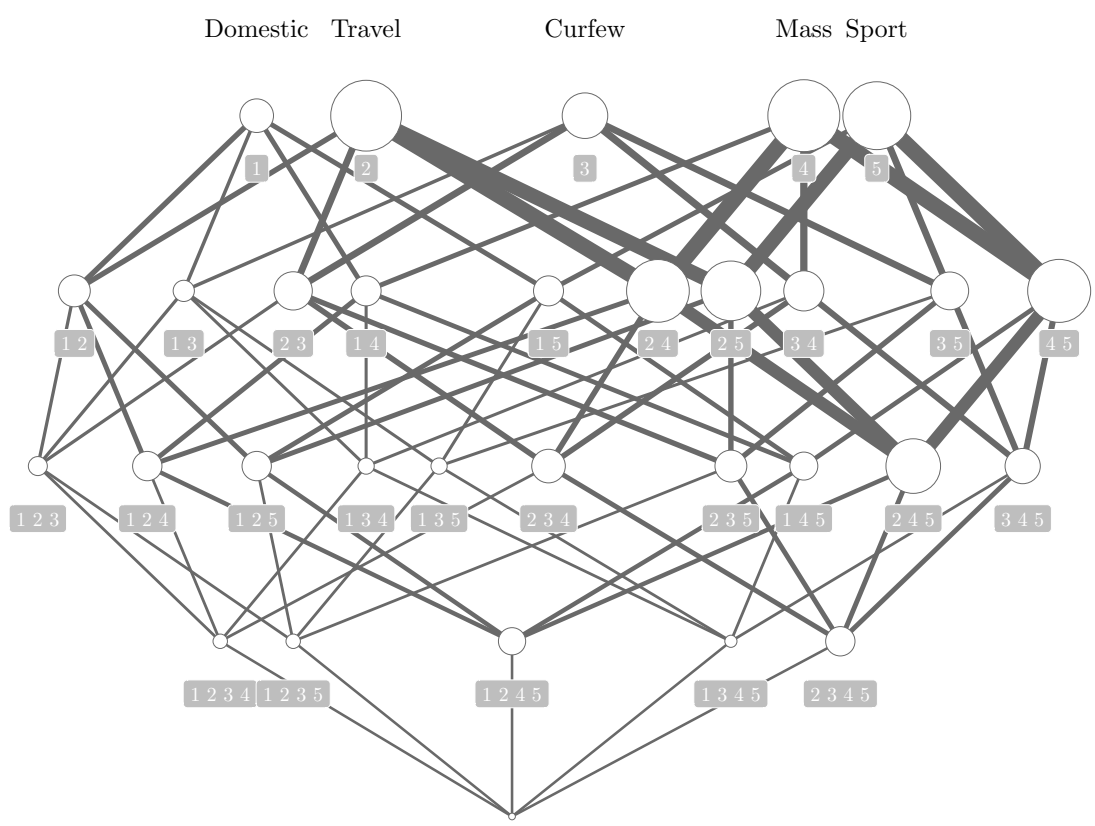

Overlap Size

Set/Intersection Size

$-25-50 \square 75 \square 100$

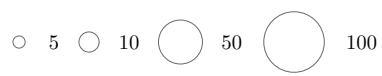


$R$ package for visualizing relational data. Also, an open-source ${ }^{1}$ web app ${ }^{2}$ is available for the effortless creation of setworks. While each of the setworks comes with its own trade-offs and features, all benefit from networks' more general powers: their detailed visualization of relational structure and their scalability. Ultimately, some of the trade-offs may be mitigated with interactive elements, such as node and edge highlights for a particular intersection depth in the chord setwork, or for a particular set and its related intersections in the waterfall setwork.

\section{References}

[1] Alexander Lex and Nils Gehlenborg. Sets and intersections. Nature Methods, 11(8):779-779, 2014.

[2] Danny Holten. Hierarchical edge bundles: Visualization of adjacency relations in hierarchical data. IEEE Transactions on Visualization and Computer Graphics, 12(5):741-748, 2006.

[3] Simon Porcher. Response2covid19, a dataset of governments' responses to COVID-19 all around the world. Scientific Data, 7(1), 2020.

[4] Kozo Sugiyama, Shojiro Tagawa, and Mitsuhiko Toda. Methods for visual understanding of hierarchical system structures. IEEE Transactions on Systems, Man, and Cybernetics, 11(2):109-125, 1981.

[5] Thomas Lin Pedersen. ggraph: An Implementation of Grammar of Graphics for Graphs and Networks, 2020. R package version 2.0.4.

1 https://github.com/aosavi/setworks

2 https://aosavi.shinyapps.io/setworks/ 